\title{
Micorrizas arbusculares no crescimento de mudas de sabiá em um substrato proveniente da mineração de manganês
}

\section{Arbuscular mycorrhiza on the growth of sabia seedlings on a substrate from the manganese mining}

\author{
Kaio Gráculo Vieira Garcia ${ }^{1 *}$, Vânia Felipe Freire Gomes ${ }^{2}$, Aldênia Mendes Mascena de Almeida ${ }^{3}$, Paulo Furtado Mendes \\ Filho
}

\begin{abstract}
Resumo: O manganês (Mn) é considerado um micronutriente essencial às plantas, porém o seu excesso pode resultar em toxidez, causando clorose e necrose nas folhas. $\mathrm{O}$ uso de leguminosas inoculadas com fungos micorrízicos arbusculares tem se tornado uma importante estratégia do ponto de vista da remediação de áreas contaminadas com metais como é o caso do manganês. Neste sentido, o objetivo deste trabalho foi avaliar o crescimento de mudas de Mimosa caesalpiniaefolia Benth. inoculadas com micorrizas arbusculares sob condições de substrato esterilizado e natural proveniente de uma área de mineração de Mn do município de Ocara - CE. O experimento foi conduzido em casa de vegetação, utilizando-se o delineamento inteiramente casualizado, em esquema fatorial 4x2, considerando-se: quatro tratamentos de inoculação (controle não-inoculado, inoculado com Glomus clarum, inoculado com Glomus etunicatum e com a mistura dos dois fungos (Mix)) e duas condições de substrato (estéril e natural), com quatro repetições. As mudas de sabiá foram produzidas em bandeja de isopor, sendo transplantadas e inoculadas em vasos após treze dias da semeadura. Após trinta dias do transplantio foram realizadas avaliações de altura da parte áerea, diâmetro do colo, número de folíolos e porcentagem de sobrevivência. A inoculação com fungos micorrízicos arbusculares favoreceu o crescimento das mudas de sabiá em substrato da mineração de manganês, em condições naturais, principalmente quando se utilizou o Glomus etunicatum. No substrato esterilizado a inoculação com micorrizas arbusculares proporcionou a atenuação da toxidez de Mn nas mudas de sabiá favorecendo o crescimento das mesmas nestas condições.
\end{abstract}

Palavras-chaves: Mimosa caesalpiniaefolia Benth, leguminosa, metais, remediação.

Abstract: Manganese (Mn) is considered an essential micronutrient to plants but its excess can result in toxicity causing chlorosis and necrosis on the leaves. The use of leguminous plants inoculated with mycorrhizal fungi have become an important point of view of the remediation strategy contaminated with metals such as manganese. In this sense, the objective of this study was to evaluate the growth of Mimosa caesalpiniaefolia Benth. seedlings inoculated with mycorrhiza under conditions of sterile and natural substrate from a Mn mining area of the municipality of Ocara - CE. The experiment was conducted in a greenhouse using a completely randomized design in a 4x2 factorial scheme considering four inoculation treatments (non-inoculated control, inoculated with Glomus clarum, inoculated with Glomus etunicatum and with the mixture of two fungi (Mix)) and two substrate conditions (sterile and natural), with four replications. The seedlings of sabiá were produced in styrofoam tray, being transplanted and inoculated in pots after thirteen days of sowing. After thirty days of transplanting been evaluated shoot height, stem diameter, number of leaflets and percentage of survival. Inoculation with mycorrhizal fungi favored the growth of sabia seedlings in manganese mining substrate, in natural conditions, especially when using the Glomus etunicatum. In the substrate sterilized inoculation with mycorrhiza provided attenuation of Mn toxicity in sabiá seedlings favoring their growth under these conditions.

Key words: Mimosa caesalpiniaefolia Benth, leguminous, metals, remediation.

\footnotetext{
*Autor para correspondência

Recebido para publicação em 10/03/2016; aprovado em 12/04/2016

${ }^{1}$ Eng. Agr., Doutorando (CAPES) do Programa de Pós-Graduação em Agronomia - Solos e Nutrição de Plantas da UFC, Fortaleza - CE, E-mail:

kaiovieira88@hotmail.com.

${ }^{2}$ Prof. (a) Dra. do Departamento de Ciências do Solo da UFC, Campus do PICI, Fortaleza - CE, E-mail: vaniafreire@ufc.br

${ }^{3}$ Bolsista de Pós-Doutorado do Departamento de Engenharia agrícola da UFC, Campus do PICI, Fortaleza - CE, E-mail: ald_m_m@ @otmail.com

${ }^{4}$ Prof. Dr. do Departamento de Ciências do Solo da UFC, Campus do PICI, Fortaleza - CE, E-mail: mendes@ufc.br
} 


\section{INTRODUÇÃO}

O manganês $(\mathrm{Mn})$ é considerado um micronutriente essencial para as plantas, o qual possui um papel de fundamental importância no metabolismo e na ativação de diferentes enzimas (LAMBERS et al. 2015). No entanto, o excesso de Mn no solo pode resultar em toxidez nas plantas (XUE et al., 2004; MIN et al., 2007; PENG et al., 2008) podendo causar manchas de coloração marrom nas folhas mais velhas, clorose e necrose, bem como o atraso no crescimento das plantas (BALDISSEROTO et al., 2007; YANG et al., 2013).

Uma série de fontes antropogênicas e naturais de Mn são consideradas principais causadoras do excesso desse elemento no solo, dentre elas a atividade de mineração. Geralmente em áreas de mineração onde ocorre a exploração deste elemento há uma intensa movimentação do solo para abertura das lavras, além da retirada da vegetação natural o qual pode causar sérias consequências ambientais (MOSSA; JAMES 2013). Ainda nesse contexto, outro ponto que deve ser chamado atenção nessas áreas é o fato da alta produção e deposição de pilhas de rejeito após o processo de beneficiamento, os quais são considerados como um dos principais fatores que causam a degradação do solo, bem como a contaminação por $\mathrm{Mn}$ e outros elementos metálicos (HAO; JIANG, 2013).

Diante desse contexto, a busca por alternativas que minimizem este tipo de impacto tem se tornado constante nos últimos anos. O uso de espécies leguminosas associadas a fungos micorrízicos arbusculares (FMA) tem se tornado uma importante estratégia do ponto de vista da remediação dessas áreas. Espécies de leguminosas geralmente são caracterizadas por possuírem uma elevada produção de biomassa com baixa relação $\mathrm{C} / \mathrm{N}$, além de um sistema radicular profundo (CAMPELO, 1998), contribuindo assim para uma melhor absorção de água e sais minerais (LINS et al., 2007). O Sabiá, Mimosa caesalpiniaefolia Benth., é uma leguminosa de porte arbóreo que tem se destacado em diversos estudos envolvendo a recuperação de áreas degradadas e contaminadas por metais pesados, principalmente em função de suas características morfológicas (MARQUES et al., 2000; CIPRIANI et al., 2013; GARCIA, 2015).

Além disso, essa espécie é capaz de se associar simbioticamente com FMA os quais podem melhorar absorção de nutrientes pelas plantas, principalmente o $\mathrm{P}$, o qual geralmente é caracterizado por baixos níveis em ambientes degradados (MARINHO et al., 2004). Ademais, estes micro-organismos podem também melhorar a absorção de água e outros elementos (SOLÍS-DOMÍNGUEZ et al., 2011), bem como a tolerância a elevadas concentrações de metais no solo, favorecendo desta forma a sobrevivência das espécies vegetais em ambientes sob estas condições (LIN et al., 2007; GOMES et al., 2014; CABRAL et al., 2015) assim como demonstrado por Nogueira et al. (2004) em plantas de soja micorrizadas em solo contaminado com manganês.

Neste sentido, o objetivo deste trabalho foi avaliar o crescimento de mudas de Mimosa caesalpiniaefolia Benth. inoculadas com diferentes fungos micorrízicos arbusculares sob condições de substrato esterilizado e natural proveniente de uma área de mineração de Mn.

\section{MATERIAL E MÉTODOS}

O experimento foi conduzido na casa de vegetação do Departamento de Ciências do Solo (DCS) da Universidade Federal do Ceará (UFC), localizada no Campus do Pici, Fortaleza, Ceará. O substrato utilizado para o crescimento das plantas foi coletado na profundidade de $0-20 \mathrm{~cm}$, em uma área de exploração mineral de manganês localizada no município de Ocara - CE.

Após a coleta foi realizado um peneiramento em malha de $4 \mathrm{~mm}$, sendo que uma parte do substrato foi esterilizada em autoclave a $121^{\circ} \mathrm{C}$ a 1 atm de pressão por duas horas e incubado durante o período de 14 dias, enquanto a outra parte não autoclavada permaneceu em condições naturais. As características químicas e físicas do substrato (antes e após a autoclavagem) encontra-se presente na Tabela 1.

Tabela 1 - Características químicas e físicas do substrato (natural e esterilizado) utilizado no experimento.

\begin{tabular}{ccc}
$\begin{array}{c}\text { Parâmetros } \\
\text { Químicos }\end{array}$ & $\begin{array}{c}\text { Substrato } \\
\text { Natural }\end{array}$ & $\begin{array}{c}\text { Substrato } \\
\text { Esterilizado }\end{array}$ \\
\hline $\mathrm{pH}\left(\mathrm{H}_{2} \mathrm{O} 1: 2,5\right)$ & 5,1 & 5,74 \\
$\mathrm{Al}(\mathrm{cmolc} / \mathrm{kg})$ & 0,5 & 0,25 \\
$\mathrm{Ca}(\mathrm{cmolc} / \mathrm{kg})$ & 1,4 & 1,7 \\
$\mathrm{Mg}(\mathrm{cmolc} / \mathrm{kg})$ & 1 & 1,4 \\
$\mathrm{~N}(\mathrm{~g} / \mathrm{kg})$ & 0,25 & 0,25 \\
$\mathrm{~K}(\mathrm{cmolc} / \mathrm{kg})$ & 0,15 & 0,18 \\
$\mathrm{P}(\mathrm{mg} / \mathrm{kg})$ & 2,9 & 4 \\
$\mathrm{Na}(\mathrm{cmolc} / \mathrm{kg})$ & 0,09 & 0,12 \\
$\mathrm{H}+\mathrm{Al}(\mathrm{cmolc} / \mathrm{kg})$ & 2,81 & 2,81 \\
$\mathrm{C}(\mathrm{g} / \mathrm{kg})$ & 2,28 & - \\
$\mathrm{M} . \mathrm{O} .(\mathrm{g} / \mathrm{kg})$ & 3,93 & - \\
$\mathrm{Mn}(\mathrm{mg} / \mathrm{kg})$ & 420 & 725,5 \\
$\mathrm{Fe}(\mathrm{mg} / \mathrm{kg})$ & 82,81 & 135,81 \\
$\mathrm{Cu}(\mathrm{mg} / \mathrm{kg})$ & 3,14 & 4,32 \\
$\mathrm{Zn}(\mathrm{mg} / \mathrm{kg})$ & 4,91 & 5 \\
\hline
\end{tabular}

\begin{tabular}{ccc}
\hline Parâmetros Físicos & & \\
\hline Areia $(\%)$ & 72,81 & - \\
Silte $(\%)$ & 16,62 & - \\
Argila $(\%)$ & 10,57 & - \\
Classificação & Franco- & - \\
Textural & arenosa & -
\end{tabular}

N (método micro-Kjeldahl); / P (Digestão nitroperclórica, colorimetria); / K, $\mathrm{Na}$ (Digestão nitroperclórica, espectrofotometria de chama);/ $\mathrm{Ca}, \mathrm{Mg}, \mathrm{Cu}$, $\mathrm{Mn}, \mathrm{Zn}$ e $\mathrm{Fe}$ (Digestão nitroperclórica, espectrofotometria de absorção atômica);/Análise granulométrica (método da pipeta - Embrapa, 1997).

Em seguida o substrato foi distribuído em vasos com capacidade para 4 litros de substrato, os quais posteriormente receberam adubação com: $27 \mathrm{mg}$ de N, $100 \mathrm{mg}$ de K, $40 \mathrm{mg}$ de $\mathrm{P}$ e $30 \mathrm{mg}$ de $\mathrm{Ca}$ por vaso. As fontes dos nutrientes utilizados foram: $\mathrm{NH}_{2} \mathrm{CONH}_{2}, \mathrm{KCl}, \mathrm{Ca}\left(\mathrm{H}_{2} \mathrm{PO}_{4}\right)_{2} \cdot \mathrm{H}_{2} \mathrm{O}$ e $\mathrm{CaSO} 4$ respectivamente.

As sementes de sabiá (Mimosa caesalpiniaefolia Benth.) foram provenientes da empresa Biosementes. As mudas foram produzidas em bandejas de isopor, colocando-se duas sementes por célula, na profundidade de $2 \mathrm{~cm}$. O substrato utilizado para produção das mudas foi areia lavada e autoclavada.

Após o estabelecimento da germinação (13 dias após a semeadura), as mudas foram inoculadas com $40 \mathrm{~g}$ de soloinóculo contendo esporos e fragmentos de raízes de milho (Zea mays L.) colonizadas pelas espécies Glomus clarum e 
Glomus etunicatum isoladamente e em mistura, por ocasião do transplantio das mudas para os vasos plásticos. O inóculo foi colocado a $4 \mathrm{~cm}$ aproximadamente, abaixo da superfície do substrato contido no recipiente. Os tratamentos com substrato autoclavado (estéril) receberam $10 \mathrm{~mL}$ de uma solução proveniente do substrato natural (não autoclavado) com concentração de $10 \mathrm{~cm}^{3}$ de solo por $1 \mathrm{dm}^{3}$ de água destilada filtrada em peneira de malha de $44 \mu \mathrm{m}$, e posteriormente filtrada em papel filtro (com a finalidade de reter os propágulos de FMA nativos), visando o reestabelecimento da comunidade microbiana.

Cada vaso recebeu duas plantas, as quais foram mantidas em casa de vegetação e irrigadas diariamente por 30 dias. Após esse período foram avaliados os parâmetros de: altura da parte aérea, diâmetro do colo, número de folíolos e porcentagem de sobrevivência.

O delineamento utilizado foi inteiramente casualizado, em esquema fatorial $4 \times 2$, considerando-se: quatro tratamentos de inoculação (controle não-inoculado, inoculado com Glomus clarum, inoculado com Glomus etunicatum e com a mistura dos dois fungos (Mix)) e duas condições de substrato (estéril e natural), com quatro repetições.

Os dados foram submetidos a análise de variância e as médias, comparadas pelo teste de Scott-Knott a $1 \%$ de probabilidade, utilizando o programa estatístico ASSISTAT versão 7.7 Beta (SILVA, 2013).

\section{RESULTADOS E DISCUSSÃO}

De acordo com a análise de variância foi observada interação significativa $(p \leq 0,01)$ entre FMA e condição de substrato para as variáveis altura, diâmetro do colo e número de folíolos. Em relação à porcentagem de sobrevivência, não houve efeito significativo em nenhuma das fontes de variação (Tabela 2).

Tabela 2 - Resumo da análise de variância para altura da parte aérea, diâmetro do colo, número de folíolos e porcentagem de sobrevivência de $M$. caesalpiniaefolia Benth. submetidas a quatro tratamentos de inoculação com FMA e duas condições de substrato (estéril e natural).

\begin{tabular}{cccc}
\hline Fontes de Variação & ALT $^{\mathrm{a}}$ & DC $^{\mathrm{b}}$ & $\mathrm{NF}^{\mathrm{c}}$ \\
\hline Tratamento de Inoculação (FMA) & $5,39^{* * *}$ & $27,95^{* *}$ & $14,26^{* *}$ \\
Condição de Substrato (CS) & $22,40^{* *}$ & $9,87^{* *}$ & 100 \\
FMA x CS & $10,40^{* *}$ & $4,85^{* *}$ & 100 \\
Coeficiente de variação (\%) & 17,13 & 9,9 & $9,51^{* *}$ \\
\hline
\end{tabular}

$\mathrm{e}^{\mathrm{ns}}$ : Significativo a 1 e $5 \%$ de probabilidade e não significativo, respectivamente.

${ }^{\mathrm{a}}$ Altura da parte aérea $(\mathrm{cm}) ;{ }^{\mathrm{b}}$ Diâmetro do colo $(\mathrm{mm}) ;{ }^{\mathrm{c}}$ Número de folíolos (und. planta ${ }^{-1}$ ); ${ }^{\mathrm{d}}$ Porcentagem de sobrevivência $(\%)$; ${ }^{1} \mathrm{FMA}$ x CS significa a interação entre FMA e CS.

É importante relatar que durante o período de condução do experimento, as plantas de $M$. caesalpiniaefolia Benth. cultivadas no substrato na condição estéril apresentaram sintomas de toxidez de manganês para o tratamento controle (Figura 2). No entanto, as plantas
No tocante à altura das plantas (Figura 1), verificou-se que na condição de substrato natural o tratamento de inoculação com Glomus etunicatum promoveu a maior média de altura $(21,06 \mathrm{~cm})$ das plantas de sabiá, obtendo-se um aumento de $15,4 \%$ quando comparado ao tratamento de inoculação controle. Enquanto que, para condição de substrato estéril o maior valor de altura $(18,81 \mathrm{~cm})$ foi verificado para o tratamento de inoculação Mix (Glomus clarum + Glomus etunicatum), o qual proporcionou um aumento de $51,3 \%$ em relação ao controle nesta mesma condição.

Figura 1. Altura da parte aérea $(\mathrm{cm})$ de $M$. caesalpiniaefolia Benth submetidas a quatro tratamentos de inoculação micorrízica sob condição de substrato estéril (autoclavado) e natural (não autoclavado).

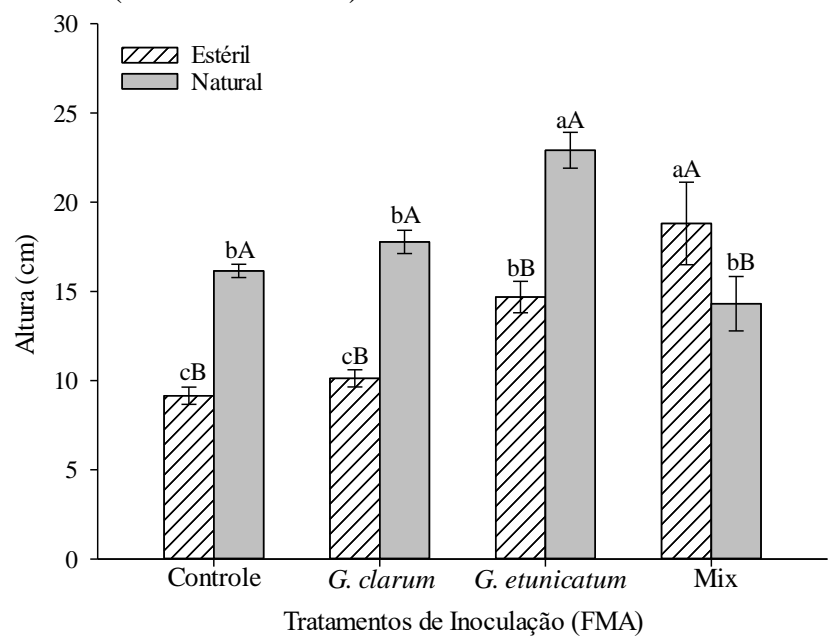

Os valores representam a média de quatro repetições \pm o erro padrão. Médias seguidas pela mesma letra maiúscula entre condições de substrato dentro de cada tratamento e minúscula entre os tratamentos de inoculação dentro de cada condição de substrato, não diferem pelo teste de Scott-Knott a $1 \%$ de probabilidade. inoculadas com Glomus clarum apesar de crescimento semelhante ao tratamento controle, não apresentaram sintomas de toxidez de $\mathrm{Mn}$ ao final dos 30 dias. Durante este tempo não foi constatado sintomas de toxidez para as plantas cultivadas em substrato na condição natural.

Figura 2. Plantas de Mimosa caesalpinieafolia Benth. cultivadas no substrato da mineração de Mn sob condição estéril submetidas a quatro tratamentos de inoculação com FMA (controle, G. clarum, G. etunicatum, mix).
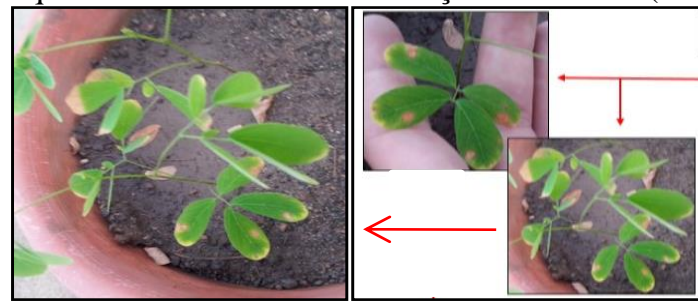

Fonte: elaborado pelos autores.

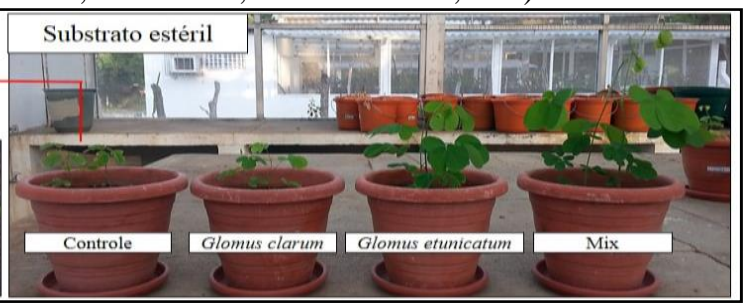

Revista Verde, v.11, n.2, p.15-20, 2016 
De acordo com Cardoso et al. (2003), a atenuação da toxidez de Mn em plantas inoculadas com FMA pode ser atribuída a um efeito indireto do maior teor de $\mathrm{P}$ nos tecidos, o que por sua vez pode complexar-se com o manganês dentro da planta diminuindo assim sua atividade. De forma geral, os maiores valores de altura foram proporcionados pela combinação da condição de substrato natural com o tratamento de inoculação Glomus etunicatum ao final dos 30 dias.

Lins et al. (2007), ao estudar o efeito de diferentes fungos micorrízicos arbusculares em solos sob impacto de mineração de cobre, observaram maior crescimento em altura das plantas cultivadas em um solo de uma área de rejeito quando inoculadas com a mistura de Glomus etunicatum + Acaulospora longula em relação ao tratamento sem inoculação. Resultados semelhantes também foram observados por Mendes et al. (2009) ao estudar o potencial de espécies florestais inoculadas com fungos micorrízicos arbusculares (FMA) em uma área degradada pela mineração de cassiterita.

Estes autores relataram um efeito significativo na altura da parte aérea da espécie Acacia mangium quando inoculada com FMA. Em um estudo mais recente, Becerril et al. (2013) ao estudarem os impactos da atividade de mineração de manganês no ambiente, observaram que plantas de Ambrosia psilostachya colonizadas por FMA diminuíram a absorção de Mn no tecido vegetal em contraste com plantas não inoculadas, resultando em um melhor desenvolvimento.

No que concerne à variável número de folíolos (Tabela 3), observa-se que plantas de $M$. caesalpiniaefolia Benth. inoculadas com FMA Glomus etunicatum, principalmente sob condição natural, apresentaram os maiores valores para o número de folíolos (51,37 und. planta $\left.{ }^{-1}\right)$, proporcionando assim um aumento de $15,5 \%$ comparado a ausência de inoculação com FMA nessa mesma condição.

Tabela 3 - Média do número de folíolos e diâmetro do colo de M. caesalpiniaefolia Benth submetidas a quatro tratamentos de inoculação com FMA e duas condições de substrato (estéril e natural).

\begin{tabular}{|c|c|c|c|c|}
\hline \multirow{2}{*}{ Condição do Substrato } & \multicolumn{4}{|c|}{ Tratamento de Inoculação } \\
\hline & Controle & Glomus clarum & Glomus etunicatum & Mix \\
\hline & \multicolumn{4}{|c|}{ Número de folíolos (und. planta $^{-1}$ ) } \\
\hline Estéril & $24,75 \pm 1,19 \mathrm{bB}$ & $27,12 \pm 0,51 \mathrm{bB}$ & $41,75 \pm 2,13 \mathrm{aB}$ & $45,62 \pm 2,13 \mathrm{aA}$ \\
\hline Natural & $43,37 \pm 4,17 \mathrm{bA}$ & $44,87 \pm 2,87 \mathrm{bA}$ & $51,37 \pm 1,70 \mathrm{aA}$ & $42,75 \pm 1,54 \mathrm{bA}$ \\
\hline Coeficiente de variação $(\%)$ & \multicolumn{4}{|c|}{11,37} \\
\hline & \multicolumn{4}{|c|}{ Diâmetro do colo (mm) } \\
\hline Estéril & $1,29 \pm 0,02 \mathrm{bB}$ & $1,32 \pm 0,02 \mathrm{bB}$ & $1,96 \pm 0,09 \mathrm{aB}$ & $1,86 \pm 0,09 \mathrm{aA}$ \\
\hline Natural & $1,66 \pm 0,07 \mathrm{bA}$ & $1,57 \pm 0,13 \mathrm{bA}$ & $2,29 \pm 0,05 \mathrm{aA}$ & $1,66 \pm 0,09 \mathrm{bA}$ \\
\hline Coeficiente de variação $(\%)$ & \multicolumn{4}{|c|}{9,9} \\
\hline
\end{tabular}

${ }^{1}$ Os valores representam a média de quatro repetições \pm erro padrão;

${ }^{2}$ Médias seguidas pela mesma letra, minúscula na linha e maiúscula na coluna, não diferem entre si pelo teste de Scott-Knott a $1 \%$ de probabilidade.

Comportamento semelhante também foi observado para o diâmetro do colo (Tabela 3) das plantas de $M$. caesalpiniaefolia Benth, no qual o tratamento com Glomus etunicatum sob condição natural proporcionou um aumento de $27,5 \%$ quando comparado a ausência de inoculação (controle).

De forma geral, as plantas de Mimosa caesalpiniaefolia Benth. inoculadas principalmente com FMA Glomus etunicatum e cultivadas sob condição natural apresentaram melhor desenvolvimento e consequentemente melhores resultados em altura da parte aérea, número de folíolos e diâmetro do colo, bem como uma maior atenuação dos sintomas de toxidez, com exceção do tratamento Mix, o qual ao analisar entre as condições de substrato não apresentou diferença significativa para o número de folíolos e diâmetro do colo.

É importante relatar que a maior redução no crescimento da espécie $M$. caesalpiniaefolia Benth. cultivada no substrato estéril comparado ao natural pode estar relacionado com o aumento de $\mathrm{Mn}$ no substrato após o procedimento de esterilização. Este aumento foi cerca de 42,1\% da condição natural para a estéril (autoclavada) (Tabela 1). De acordo com Nogueira et al. (2003) o aumento na disponibilidade de manganês após autoclavagem pode ser atribuído a quebra térmica dos quelantes orgânicos, um dos principais fatores que regulam a disponibilidade de $\mathrm{Mn}$.

$\mathrm{O}$ manganês $(\mathrm{Mn})$ é um micronutriente essencial para as plantas, no entanto, o excesso deste elemento pode torna-se prejudicial ao desenvolvimento das plantas. De acordo com alguns autores (FOY, 1984; MORONI et al., 2003; WEI et al., 2014) a toxidade por manganês é um dos fatores mais limitantes ao crescimento de espécies vegetais, principalmente em grande parte dos solos ácidos no mundo. Ainda nesse contexto, Cardoso et al. (2002) relatam que o excesso de metais, entre eles o Mn, pode diminuir ou inibir o desenvolvimento inicial de hifas fúngicas e consequentemente atrasar ou suprimir a formação de micorriza.

Em geral, estudos com fungos micorrízicos arbusculares no crescimento de espécies vegetais em áreas de mineração, como no caso do Mn ainda são bastante escassos. Neste sentido, o conhecimento de um determinado FMA que proporcione melhores resultados em termos de crescimento e consequentemente tolerância a uma determinada espécie vegetal em áreas como a desse estudo, poderá ser utilizado para nortear trabalhos que visem a recuperação de ambientes com alta disponibilidade de $\mathrm{Mn}$.

\section{CONCLUSÕES}

A inoculação com fungos micorrízicos arbusculares favoreceu o crescimento de mudas de Mimosa caesalpiniaefolia Benth. em substrato da mineração de manganês, em condições naturais, principalmente quando se utilizou o Glomus etunicatum.

No substrato estéril (autoclavado) a inoculação com fungos micorrízicos arbusculares proporcionou a atenuação da toxidez de Mn em mudas de Mimosa caesalpiniaefolia 
Benth. favorecendo o crescimento das mesmas nestas condições.

\section{REFERÊNCIAS BIBLIOGRÁFICAS}

BALDISSEROTTO, C.; FERRONI, L.; ANFUSO, E.; PAGNONI, A.; FASULO, M.P.; PANCALDI, S. Responses of Trapa natans L. Floating laminae to high concentrations of manganese. Protoplasma, v.231, n.1-2, p.65-82, 2007.

BECERRIL, F. R.; VAZQUEZ, L. V. J.; CERVANTES, S. C. H.; SANDOVAL, O. A. A.; CORREA, G. V.; CHAVEZ, E. C.; ESPINDOLA, I. P. M.; HERRERA, A. E.; GONZALEZ, F. L. Impacts of manganese mining activity on the environment: interactions among soil, plants, and arbuscular mycorrhiza. Archives of Environmental Contamination and Toxicology, v.64, p.219-227, 2013.

CABRAL, L.; SOARES, C. R. F. S.; GIACHINI, A. J.; SIQUEIRA, J. O. Arbuscular mycorrhizal fungi in phytoremediation of contaminated areas by trace elements: mechanisms and major benefits of their applications. World Journal of Microbiology and Biotechnology, v.31, n.11, p.1655-1664, 2015.

CAMPELLO, E. F. C. Sucessão vegetal na recuperação de áreas degradadas. In: DIAS, L. E.; MELLO, J. W. V. (Ed.). Recuperação de Áreas Degradadas. Viçosa, MG: Universidade Federal de Viçosa / Sociedade Brasileira de Recuperação de Áreas Degradadas, 1998. p.183-196.

CARDOSO, E. J. B. N.; NAVARRO, R. B.; NOGUEIRA, M. A. Absorção e translocação de manganês por plantas de soja micorrizadas, sob doses crescentes deste nutriente. Revista Brasileira de Ciência do Solo, v. 27, n. 3, p.415-423, 2003.

CARDOSO, E. J. B. N.; NAVARRO, R. B.; NOGUEIRA, M. A. Manganês e germinação de esporos de fungos micorrízicos arbusculares in vitro. Revista Brasileira de Ciência do Solo, v. 26, p.795-799, 2002.

CIPRIANI, H. N.; DIAS, L. E.; COSTA, M. D.; CAMPOS, N. V.; AZEVEDO, A. A.; GOMES, R. J.; FIALHO, I. F.; AMEZQUITA, S. P. M. Arsenic toxicity in Acacia mangium willd. and Mimosa Caesalpiniaefolia Benth. seedlings. Revista Brasileira de Ciência do Solo, v. 37, p. 1423-1430, 2013.

EMBRAPA. Centro Nacional de Pesquisa de Solos. Manual de métodos de análise de solos. 2 ed. rev. e atual. Rio de Janeiro: EMBRAPA, 1997.212p.

FOY, C. D. Physiological effects of hydrogen, aluminium and manganese toxi- cities in acid soils, in soil acidity and liming. Agron. Mon. 12, 57-97, 1984.

GARCIA, K. G. V. Desenvolvimento de Mimosa caesalpiniaefolia Benth. colonizada com micorrizas arbusculares em solos degradados por mineração de $\mathrm{Mn}$. 2015. 66f. Dissertação (Mestrado em Agronomia - Solos e Nutrição de Plantas) - Universidade Federal do Ceará, Fortaleza. 2015.
GOMES, M. P.; ANDRADE, M. L.; NASCENTES, C. C.; SCOTTI, M. R. Arsenic Root Sequestration by a Tropical Woody Legume as Affected by Arbuscular Mycorrhizal Fungi and Organic Matter: Implications for Land Reclamation. Water, Air, \& Soil Pollution, v.225, p.1-12, 2014.

HAO, Q.; JIANG, C. Heavy metal concentrations in soils and plants in Rongxi Manganese Mine of Chongqing, Southwest of China. Acta Ecologica Sinica, v.35, n.1, p.46-51, 2015.

LAMBERS, H.; HAYES, P. E.; LALIBERTE, E.; OLIVEIRA, R. S.; TURNER, B. L. Leaf manganese accumulation and phosphorus-acquisition efficiency. Planta Science, v.20, n.2, p.83-90, 2015.

LIN, A.; ZHANG, X.; WONG, M.; YE，Z.; LOU, L.; WHANG, Y.; ZHU, Y. Increase of multi-metal tolerance of three leguminous plants by arbuscular mycorrhizal fungi colonization. Environmental Geochemistry and Health, v.29, n.6, p. 473-481, 2007.

LINS, C. E. de L.; MAIA, L. C.; CAVALCANTE, U. M. T.; SAMPAIO, E. V. de S. B. Efeito de fungos micorrízicos arbusculares no crescimento de mudas de Leucaena leucocephala (LAM.) de wit. em solos de caatinga sob impacto de mineração de cobre. Revista Árvore, v.31, n.2, p.355-363, 2007.

MARINHO, N. F.; CAPRONI, A. L.; FRANCO, A. A.; BERBARA, R. L. L. Respostas de Acacia mangium Willd e Sclerolobium paniculatum Vogel a fungos micorrízicos arbusculares nativos provenientes de áreas degradadas pela mineração de bauxita na Amazônia. Acta Botanica Brasilica, v.18, n.1, p. 141-149, 2004.

MARQUES, T. C. L. L. de S. M.; MOREIRA, F. M. de S.; SIQUEIRA, J. O. Crescimento e teor de metais de mudas de espécies arbóreas cultivadas em solo contaminado com metais pesados. Pesquisa Agropecuário Brasileira, v.35, n.1, p. 121$132,2000$.

MENDES FILHO, P. F.; VASCONCELLOS, R. L. F.; PAULA, A. M. de; CARDOSO, E. J. B. N. Evaluating the potential of forest species under "microbial management" for the restoration of degraded mining áreas. Water, Air, \& Soil Pollution, v. 208, n. 1-4, p. 79-89, 2009.

MIN, Y.; BOQING, T.; MEIZHEN, T.; AOYAMA, I. Accumulation and uptake of manganese in hyperaccumulator Phitolacca americana. Minerals Engineering, v.20, n.2, p.188-190, 2007.

MORONI, J. S.; SCOTT, B. J.; WRATTEN, N. Differential tolerance of high manganese among rapeseed genotypes. Plant Soil, v.253, p.507-519, 2003.

MOSSA, J.; JAMES, L. A. Impacts of mining on geomorphic systems. In: SHRODER, J. (Editor in chief), JAMES, L.A., HARDEN, C.P., CLAGUE, J.J. (Eds.), Treatise on Geomorphology. Academic Press, San Diego, CA, vol. 13, Geomorphology of Human Disturbances, Climate Change, and Natural Hazards, p. 74-95, 2013. 
NOGUEIRA, M. A.; CARDOSO, E. J. B. N. Mycorrhizal effectiveness and manganese toxicity in soybean as affected by soil type and endophyte. Scientia Agricola, v. 60, n. 2, p.329-335, 2003.

NOGUEIRA, M. A.; MAGALHÃES, G. C.; CARDOSO, E. J. B. N. Manganese toxicity in mycorrhizal and phosphorusfertilized soybean plants. Journal of Plant Nutrition, v. 27, n. 1, p. 141-156, 2004.

PENG, K.; LUO, C.; YOU, W.; LIAN, C.; LI, X.; SHEN, Z. Manganese uptake and interactions with cadmium in the hyperaccumulator. Journal of Hazardous Materials, v.154, n.1-3, p.674-681, 2008.

SILVA, F. A. S. Assistat versão 7.7 beta, distribuição gratuita. 2013.

SOLÍS-DOMÍNGUEZ，F. A.; VALENTÍN-VARGAS，A.; CHOROVER, J.; MAIER, R. M. Effect of arbuscular mycorrhizal fungi on plant biomass and the rhizosphere microbial community structure of mesquite grown in acidic lead/zinc mine tailings. Science of the Total Environment, v. 409, n. 6, p. 1009-1016, 2011.

WEI, Y.; HOU, H.; LI, J.; SHANGGUAN, Y.; XU, Y.; ZHANG, J.; ZHAO, L.; WANG, W. Molecular diversity of arbuscular mycorrhizal fungi associated with an Mn hyperaccumulator-Phytolacca americana, in Mn mining area. Applied Soil Ecology, v.82, p.11-17, 2014.

XUE, S. G.; CHEN, Y. X.; REEVES, R. D.; BAKER, A. J., LIN, Q.; FERNANDO, D. R. Manganese uptake and accumulation by the hyperaccumulator plant Phytolacca acinosa Roxb. (Phytolaccaceae). Environmental Pollution, v.131, n. 3, p.393-399, 2004.

YANG, Q.; ZENG, Q.; XIAO, F.; LIU, X.; PAN, J.; HE, J.; LI, Z. Investigation of manganese tolerance and accumulation of two Mn hyperaccumulators Phytolacca americana L. and Polygonum hydropiper L. in the real Mn-contaminated soils near a manganese mine. Environmental Earth Sciences, v.68, n.4, p.1127-1134, 2013. 